\title{
Acoustofluidics 10: Scaling laws in acoustophoresis
}

\section{Bruus, Henrik}

\section{Published in:}

Lab on a Chip

Link to article, DOI:

$10.1039 / \mathrm{c} 2 \mathrm{lc} 21261 \mathrm{~g}$

Publication date:

2012

\section{Document Version}

Peer reviewed version

Link back to DTU Orbit

\section{Citation (APA):}

Bruus, H. (2012). Acoustofluidics 10: Scaling laws in acoustophoresis. Lab on a Chip, 12(9), 1578-1586.

https://doi.org/10.1039/c2lc21261g

\section{General rights}

Copyright and moral rights for the publications made accessible in the public portal are retained by the authors and/or other copyright owners and it is a condition of accessing publications that users recognise and abide by the legal requirements associated with these rights.

- Users may download and print one copy of any publication from the public portal for the purpose of private study or research.

- You may not further distribute the material or use it for any profit-making activity or commercial gain

- You may freely distribute the URL identifying the publication in the public portal

If you believe that this document breaches copyright please contact us providing details, and we will remove access to the work immediately and investigate your claim 


\title{
Acoustofluidics 10: Scaling laws in acoustophoresis
}

\author{
Henrik Bruus
}

DOI: $10.1039 / c 2 l c 21261 \mathrm{~g}$

\begin{abstract}
In Part 10 of the thematic tutorial series "Acoustofluidics - exploiting ultrasonic standing waves forces and acoustic streaming in microfluidic systems for cell and particle manipulation", we present and analyze a number of scaling laws relevant for microsystem acoustophoresis. Such laws are useful both in understanding, designing, and analyzing acoustofluidic devices.
\end{abstract}

\section{Introduction}

In physics, scaling laws are statements about how dependent variables scale with the independent variables, e.g. the flow rate $Q$ in a microfluidic system scales like the pressure drop $\Delta p$ to the power one, written as $Q \propto \Delta p$, and the speed of sound $c_{0}$ in a liquid scales like the density $\rho_{0}$ of the liquid to the power minus one-half, written as $c_{0} \propto\left(\rho_{0}\right)^{-\frac{1}{2}}$. Scaling laws are helpful in gaining a basic physical understanding of a given system, and they are intimately related to dimensional analysis and to the concept of characteristic dimensionless groups or numbers, such as the Reynolds number.

Department of Micro- and Nanotechnology, Technical University of Denmark, DTU

Nanotech, Building 345 B, DK-2800 Kongens Lyngby,Denmark.E-mail: Henrik.Bruus@, nanotech.dtu.dk
In the following we shall see examples of scaling laws relevant for acoustofluidics and also identify important dimensionless groups, often derived by dimensional analysis combined with physical insight. In line with the context of this tutorial series, we shall see how the scaling laws are useful for understanding, designing, and analyzing acoustofluidic devices.

The treatment is divided into four sections covering (i) laminar flow in microfluidic channels, (ii) molecular diffusion in laminar flow streams, (iii) ultrasound acoustics, and (iv) motion of microparticles.

\section{Laminar flow}

As a first example of scaling laws and dimensional analysis we take the Poiseuille flow, analyzed in Part 1 of the tutorial series ${ }^{1}$ and sketched in Fig. 1.

\section{A. Dimensional analysis of the flow rate}

Let us ask the question of how the flow rate $Q$ scales with the physical parameters of the problem: pressure drop $\Delta p$, channel length $L$, cross sectional dimensions (radius $a$ for a circle, and width $w$ and height $h$ for parallel plates), as well as density $\rho_{0}$ and viscosity $\eta$ of the liquid. We know the full answer to be ${ }^{1}$

$$
\begin{gathered}
Q=\frac{\pi a^{4}}{8 \eta L} \Delta p, \quad \text { circular tube, } \\
Q=\frac{h^{3} w}{12 \eta L} \Delta p, \quad \text { parallel-plates, }
\end{gathered}
$$

but how can we obtain this from dimensional analysis?

First we list the SI units (marked by square brackets) of the involved quantities: $[Q]=\mathrm{m}^{3} \mathrm{~s}^{-1},[\Delta p]=\mathrm{Pa},[\eta]=\mathrm{Pa} \mathrm{s},[L]=[h]$ $=[w]=[a]=\mathrm{m}$. We leave out $\rho_{0}$, as densityrelated inertial effects are negligible in microfluidics systems. ${ }^{1}$ Then we look at the driving force, here the pressure drop $\Delta p$, and note that in the linear regime, the flow resistance ensures that flow rate scales like $Q \propto \Delta p$. Since the left-hand side contains 1/ $\mathrm{s}$, we must divide the right-hand side by $\eta$, the only remaining quantity containing the unit of time, $Q \propto \Delta p / \eta$. As a result, the right-hand side needs to be multiplied by some length $\ell$ to the power 3 to balance the units, $Q \propto \ell^{3} \Delta p / \eta$. But which physical lengths enters $\ell^{3}$ and to which power? Here we must go beyond the dimensional analysis and bring in some physical insight. Clearly, the longer the channel, the smaller a flow rate for a given pressure drop $\Delta p$, so

\section{Foreword}

In this tenth paper of 23 in the Lab on a chip tutorial series of Acoustofluidics, Henrik Bruus discuss scaling laws in acoustophoresis. Examples are of how different phenomena and forces balance each other in terms of laminar flow, molecular diffusion, ultrasound acoustics and micro channel acoustophoresis are presented. Dimensional analysis is a powerful tool and provides important insights of parameters to be considered when designing an acoustofluidic system.

Andreas Lenshof-coordinator of the Acoustofluidics series 


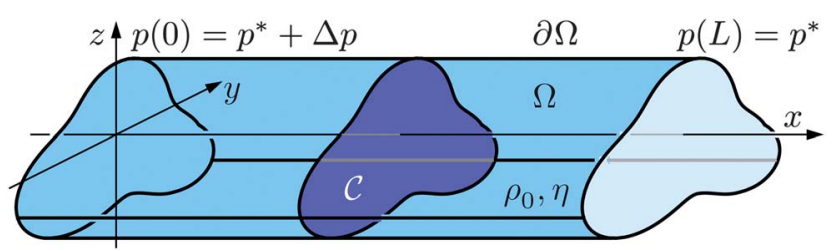

Fig. 1 Poiseuille flow of a liquid with density $\rho_{0}$ and viscosity $\eta$ through a straight channel $\Omega$ (blue), where the flow is subject to the no-slip boundary condition on the surface $\partial \Omega$. The channel is translational invariant in the $x$ direction, and it has an arbitrarily shaped cross-section $\mathscr{C}$ (dark blue) in the $y z$-plane. The pressure at $x=0$ (left) is $\Delta p$ higher than at $x=L$ (right).

for a linear response we get $Q \propto \ell^{4} \Delta p /(\eta L)$, where the length scale $\ell^{4}$ now only involves the cross-sectional geometry. Since $Q \propto$ $v \mathscr{A}$, where $v$ is the average flow velocity and $\mathscr{A}$ the area of the cross section, we have

$$
Q \propto \frac{\ell^{2} \mathscr{A}}{\eta L} \Delta p
$$

where $\ell$ is now solely related to the scaling of $v$ with the cross-sectional geometry. From the general nature of viscous flows, we know that $\ell$ must be associated with the smallest distance from the center of the channel to the side walls, as this determines the shear stress $\eta v / \ell$ dominating the flow resistance. The scaling law eqn (2) is how far we can come from a purely dimensional analysis of the Poiseuille flow for a given constant pressure $\Delta p$. A circular tube of radius $a$ will have $\ell=a$ and $\mathscr{A}=\pi a^{2}$, while a parallelplate channel with $h<w$ will have $\ell=h / 2$ and $\mathscr{A}=w h$, whereby we nearly recover the exact result eqn (1a) and (1b), except for the numerical factors $1 / 8$ and $1 / 3$, respectively. Without solving any differential equations, we can infer the expected proportionality $Q \propto \Delta p /(\eta L)$, and the less obvious, but experimentally very important, dependence of $Q$ on the smallest length scale of the cross-section, $Q \propto a^{4}$ (circle) and $Q \propto h^{3}$ (parallel plate). A linear down-scaling of a channel cross section by a factor of 2 thus reduces the flow rate by a factor of 16 for the circular pipe and by 8 for a parallel-plate channel.

\section{B. Characteristic dimensionless numbers}

The above example shows the problems that occurs when dimensionless groups or numbers appear, e.g. the length ratio $/ / L$. Dimensional analysis alone cannot determine to which power it enters the final expression. It is therefore important firstly to identify the dimensionless numbers, and secondly to determine how the sought relation depends on them.

A formal way to determine the number $\mathscr{N}$ of dimensionless groups is by using the so-called Buckingham $\pi$-theorem, ${ }^{2}$ stating that

$$
\mathscr{N}=\mathscr{D}-\mathscr{\mathscr { F }},
$$

where $\mathscr{D}$ is the number of dependent variables in the given problem, and $\mathscr{F}$ is the number of independent, fundamental

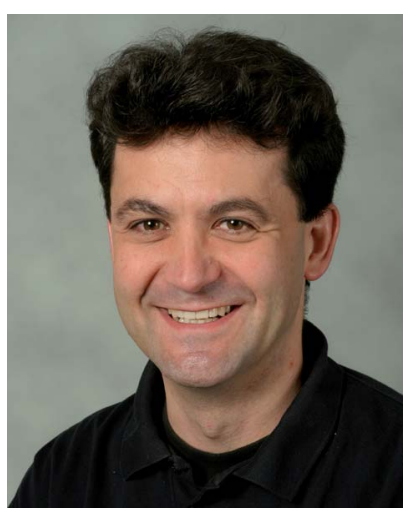

Henrik Bruus
Prof. Henrik Bruus received his B.Sc. in mathematics and physics from the University of Copenhagen in 1984 and his M.Sc. and Ph.D. degrees in physics from the Niels Bohr Institute, University of Copenhagen in 1986 and 1990, respectively. He was postdoctoral fellow at Nordic Institute of Theoretical Physics 1990-92, Yale University 1992-94 and CNRS Grenoble 1994-96. He returned to the Niels Bohr Institute as an associate professor 1997-2001, before he joined the faculty at DTU Nanotech, Technical University of Denmark in 2002. He was promoted to full professor there in 2005. He has (co)authored more than 100 peer-reviewed journal papers on condensed matter physics and microfluidics, as well as 120 peer-reviewed conference contributions and 2 monographs, the latest being "Theoretical Microfluidics“, Oxford University Press (2008). dimensions ( 3 in mechanics: length $L$, time $T$, and mass $M$ ). Let us apply the theorem for the Poiseuille flow in a circular tube described by $\mathscr{D}=6$ dependent variables, $a, v, \Delta p, \eta, \rho_{0}$, and $L$. According to Buckingham's theorem, this results in $\mathcal{N}=6-3=3$ dimensionless numbers. Buckingham also provided a method to determine them. ${ }^{2}$ The first step is to resolve the physical dimension of the $\mathscr{D}$ dependent variables $d_{k} \sim L^{\alpha_{1 k}} T^{\alpha_{2 k}} M^{\alpha_{3 k}}(k=1,2, \ldots, \mathscr{D})$ in powers of the $\mathscr{F}$ fundamental dimensions (with index $j=1,2, \ldots, \mathscr{F}$ ), such as in $[\Delta p]$ $=\mathrm{Pa}=\mathrm{kg} /\left(\mathrm{s}^{2} \mathrm{~m}\right) \sim L^{-1} T^{-2} M^{1}$. Then we construct the $\mathscr{\mathscr { F }} \times \mathscr{D}$ matrix $\mathbf{P}$ with elements $(\mathbf{P})_{j k}=\alpha_{j k}$, which in our case becomes

$$
\mathbf{P}=\left(\begin{array}{ccccccc} 
& a & v & \rho_{0} & \eta & \Delta p & L \\
\mathrm{~L} & 1 & 1 & -3 & -1 & -1 & 1 \\
\mathrm{~T} & 0 & -1 & 0 & -1 & -2 & 0 \\
\mathrm{M} & 0 & 0 & 1 & 1 & 1 & 0
\end{array}\right),
$$

where for clarity the row and column labels are shown. The dimensionless groups or numbers can now be determined as the $\mathcal{N}$ null vectors $\pi_{\mathrm{i}}$ (here $\pi_{1}$, $\pi_{2}$, and $\pi_{3}$ ) to the matrix $\mathbf{P}$,

$$
\mathbf{P} \cdot \boldsymbol{\pi}_{i}=0, \text { for } i=1,2, \ldots, \mathcal{N},
$$

because the components of these vectors contains the powers $\beta_{j k}$ of the dependent variables $d_{k}$ leading to the power zero of fundamental $j$ th dimension, $\left[d_{1}^{\beta_{j 1}} d_{2}^{\beta_{j 2}} \cdots d_{\mathscr{D}}^{\beta_{j g}}\right]=0$. Three possible dimensionless groups for the matrix in eqn (4) are

$\boldsymbol{\pi}_{1}=\left(\begin{array}{c}1 \\ 1 \\ 1 \\ -1 \\ 0 \\ 0\end{array}\right), \boldsymbol{\pi}_{2}=\left(\begin{array}{c}1 \\ 0 \\ 0 \\ 0 \\ 0 \\ -1\end{array}\right), \boldsymbol{\pi}_{3}=\left(\begin{array}{c}-1 \\ 1 \\ 0 \\ 1 \\ -1 \\ 0\end{array}\right)$,

corresponding respectively to the dimensionless groups or numbers

$$
N_{1}=\frac{a v \rho_{0}}{\eta}, \quad N_{2}=\frac{a}{L}, N_{3}=\frac{\eta v}{a \Delta p} .
$$

The first of these numbers is the Reynolds number ${ }^{1} R e$, the second is the aspect ratio of the channel, and the third is ratio of the shear-induced stress $\eta(v / a)$ and the driving pressure $\Delta p$. The values of these respective numbers reveal information about the physical state of the system. It is only if the Reynolds number is small, $R e=N_{1} \lesssim 1$, 
that it makes sense to exclude the density as a relevant parameter in the dimensional analysis in Section II A. Likewise, it is only if the channel aspect ratio is small, $N_{2} \lesssim 1$, that we can determine $\ell^{2}$ in eqn (2). Consequently, the identification of the dimensionless numbers is an important part of dimensional analysis.

An important caveat regarding the dimensionless numbers is that they are not uniquely determined. Any linear combination of the null vectors $\pi_{i}$ results in a null vector. The following set is also a possible solution,

$\boldsymbol{\pi}_{1}^{*}=\left(\begin{array}{c}1 \\ 1 \\ 1 \\ -1 \\ 0 \\ 0\end{array}\right), \boldsymbol{\pi}_{2}^{*}=\left(\begin{array}{c}-1 \\ 0 \\ 0 \\ 0 \\ 0 \\ 1\end{array}\right), \boldsymbol{\pi}_{3}^{*}=\left(\begin{array}{c}0 \\ 2 \\ 1 \\ 0 \\ -1 \\ 0\end{array}\right)$,

corresponding respectively to the dimensionless groups or numbers

$$
N_{1}^{*}=\frac{a v \rho_{0}}{\eta}, \quad N_{2}^{*}=\frac{L}{a}, \quad N_{3}^{*}=\frac{\rho_{0} v^{2}}{\Delta p} .
$$

Here $N_{1}^{*}=N_{1}$ is the Reynolds number $R e$ as before, $N_{2}^{*}=1 / N_{2}$ is the inverse aspect ratio of the channel, while $N_{3}^{*}=N_{1} N_{3}$ is the ratio of the kinetic pressure to the driving pressure. For very low flow velocities, $N_{3}^{*} \ll N_{3}$ and the shear stress dominates over the kinetic pressure. For higher velocities the situation reverses, as is of course evident from the relationship $N_{3}^{*} / N_{3}=R e$.

\section{Entrance length}

In acoustofluidics, one is often in a situation where several flow streams are brought together at the entrance of the region (here a long straight channel) of the microfluidic chip where the active acousto-activated particle separation takes place. The question naturally arises at which distance $\mathscr{L}$ down-stream from the entrance point is the laminar flow a fully developed steady-state Poiseuille flow profile.

For very low flow velocities, it is expected that the entrance length $\mathscr{L}$ scales like the smallest distance $l$ from the center of the channel to the side walls in the cross-sectional geometry ( $a$ for the circle and $h / 2$ for the parallel-plate with $h$ $<w), \mathscr{L} \propto \ell$. This must be so, as $\ell$ in this case is the only length scale in the problem. However, as the velocity
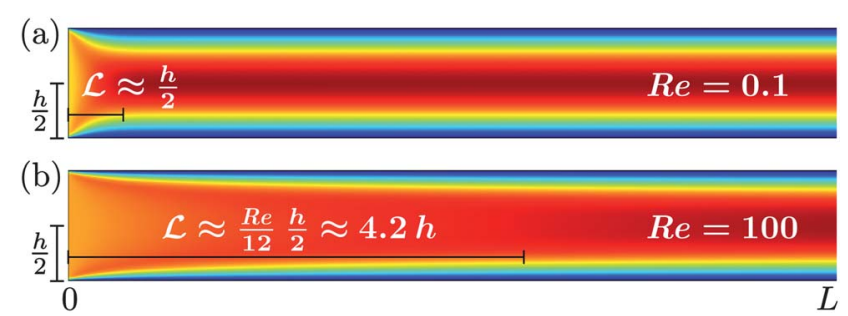

Fig. 2 Color plots of the axial velocity from zero (dark blue) to $v_{\max }$ (dark red) in a parallel-plate channel (side view) of length $L$ and height $h$. The velocity field on the inlet to the left is set to be a constant. (a) In the low Reynolds number limit $(R e=0.1)$ the entrance length over which a full Poiseuille flow profile is established is given by $\mathscr{L} \approx(1 / 2) h$. (b) In the medium Reynolds number limit $(R e=100)$ the entrance length is given by $\mathscr{L} \approx(R e / 24) h$.

is increased and the Reynolds number exceeds unity, another (kinematic) length scale appears, namely $\mathscr{L} \propto$ Re $\ell$.

As in Section II, a more physical insight is needed to obtain a more precise statement, so let us consider a simplified situation, where instead of several flow streams meeting at the inlet of the channel, we just assume that the velocity profile differs from the fully developed Poiseuille profile and is, say, the constant $v$ across the inlet. Due to mass conservation in the long straight channel we are analyzing, the average velocity remains $v$. In a reference frame where the channel walls are fixed, the steady-state flow profile has a spatial development, it simply changes its shape as a function of axial position $x$. In a reference system moving with the average, constant flow speed $v$, the development of the flow profile is temporal and not spatial. It can be analyzed using the Navier-Stokes equation in the form of the velocity diffusion equation $^{1} \partial_{t} \boldsymbol{v}=\nu \nabla^{2} \boldsymbol{v}$, where $\nu=\eta / \rho_{0}$ is the momentum diffusivity or kinematic viscosity, which for water is $v=10^{-6} \mathrm{~m}^{2}$ $\mathrm{s}^{-1}$. Diffusion length (here the shortest, transverse channel dimension $\ell$ ) and time $\tau$ is described by the exponential factor $\exp \left[-\iota^{2} /(2 v \tau)\right]$ in one spatial dimension as studied in the following section. If we define the full development of the flow profile as when the developing profile is within $5 \%$ of the steady-state value, then $\exp \left[-\ell^{2} /(2 v \tau)\right] \approx 0.05$ or $\ell^{2} \approx 6 v \tau$. The entrance length $\mathscr{L}$ in the fixed frame of reference can therefore be estimated as $\mathscr{L} \approx v \tau \approx \iota^{2} v /(6 v)=\frac{1}{12} \operatorname{Re} \iota$, where we use the usual definition of the Reynolds number $R e=(2 \iota) v \rho_{0} / \eta$. In summary, our scaling law for the entrance length in straight-channel Poiseuille flow becomes

$$
\mathscr{L} \approx \max \left\{\iota, \frac{R e}{12} \iota\right\} .
$$

In Fig. 2 is shown a numerical simulation of the entrance effects for the axial velocity field in a straight parallel plate channel of height $h$. The inlet velocity profile is the constant $v$ in the axial direction at $x=0$. In this case $\ell=h / 2$, and in panel (a) it is seen how $\mathscr{L} \approx h / 2$ for the low Reynolds number $R e=0.1$, while $\mathscr{L} \approx(\operatorname{Re} / 12) h / 2=0.042 \operatorname{Re} h=4.2 h$ for the medium Reynolds number $R e=100$ in accordance with eqn (10). We note that for typical microchannels used in acoustophoresis with $h=0.2 \mathrm{~mm}$, the Reynolds number $R e=12$ for which the scaling changes from one behavior to another in eqn (10), corresponds to the relatively high average velocity $v \approx 6 \mathrm{~cm} \mathrm{~s}^{-1}$. We therefore expect that the entrance length in acoustofluidics in most cases is given by half the channel height, $\mathscr{L} \approx \iota$, for $h<w$.

\section{Inertial time scale}

In spite of the dominance of viscosity in microfluidics systems, it can nevertheless be of interest to determine the time scale of inertial effects of the liquid flow. In Part 1 of the Tutorial Series ${ }^{1}$ this was briefly treated in terms of hydraulic inductance. As an example we study here the inertial-related time, $\tau_{\text {iner, }}$, it takes a liquid with density $\rho_{0}$, viscosity $\eta$, and kinematic viscosity $v=\eta / \rho_{0}$, to reach rest after an instantaneous removal of the pressure difference, driving a steady-state Poiseuille flow through a circular microchannel of radius $a$ and length $L$. Here "instantaneous" of course refers to the 


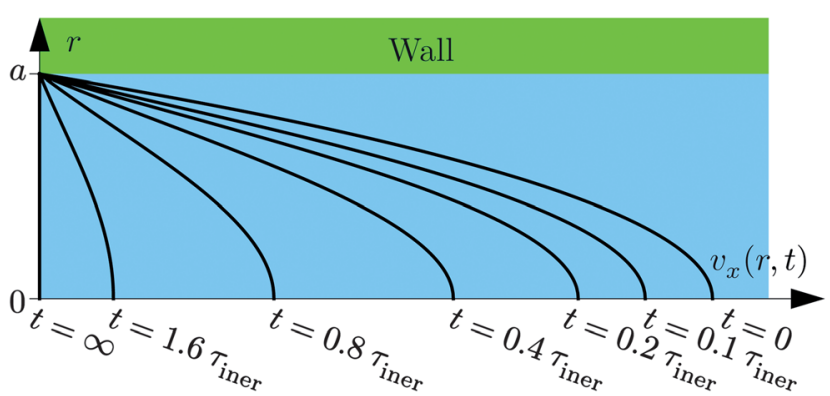

Fig. 3 The evolution in time from the initial Poiseuille paraboloid at $t=0$ to a zero velocity profile at $t=\infty$ of the velocity profile $v_{x}(r, t)$ in a cylindrical channel of radius $a$ for a decelerating Poiseuille flow due to the abrupt disappearance of the driving pressure $\Delta p$ at $t=0$. The time is expressed in units of the inertial time scale $\tau_{\text {iner }}=a^{2} /(4 v)$ of eqn (14). Adapted from ref. 3 .

small acoustic time scale $\tau_{\text {acou }}=L / c_{0}$ of pressure (or sound) wave propagation along the channel given the speed of sound $c_{0}$ of the liquid.

For this problem the Buckingham $\mathbf{P}$ matrix becomes

$\mathbf{P}=\left(\begin{array}{ccccccc} & a & L & \tau_{\text {iner }} & c_{0} & \eta & \rho_{0} \\ \mathrm{~L} & 1 & 1 & 0 & 1 & -1 & -3 \\ \mathrm{~T} & 0 & 0 & 1 & -1 & -1 & 0 \\ \mathrm{M} & 0 & 0 & 0 & 0 & 1 & 1\end{array}\right)$

with the set of $\mathscr{N}=\mathscr{D}-\mathscr{F}=6-3=3$ null vectors,

$\boldsymbol{\pi}_{1}=\left(\begin{array}{c}1 \\ -1 \\ 0 \\ 0 \\ 0 \\ 0\end{array}\right), \boldsymbol{\pi}_{2}=\left(\begin{array}{c}0 \\ 1 \\ -1 \\ -1 \\ 0 \\ 0\end{array}\right), \boldsymbol{\pi}_{3}=\left(\begin{array}{c}2 \\ 0 \\ -1 \\ 0 \\ -1 \\ 1\end{array}\right)$,

corresponding to the dimensionless groups or numbers

$$
N_{1}=\frac{a}{L}, \quad N_{2}=\frac{\tau_{\text {acou }}}{\tau_{\text {iner }}}, \quad N_{3}=\frac{a^{2}}{\nu \tau_{\text {iner }}} .
$$

The first of these is the aspect ratio of the channel, the second is the ratio of the axial propagation time of pressure waves relative to the stopping time, while the last is the ratio of the momentum diffusion time relative to the stopping time. In the case of a long, narrow channel $\left(N_{1} \ll 1\right)$, and a propagation time of sound much shorter than the stopping time $\left(N_{2} \ll 1\right)$, the liquid flow stops due to the sidewards diffusion of momentum over the distance $a$, with the momentum diffusivity $\nu$. Dimensional analysis thus leads to $\tau_{\text {iner }} \approx$ $a^{2} / v$, while standard diffusion theory for two spatial dimensions provides another factor of $1 / 4$. We therefore arrive at the scaling law for the inertial stopping time,

$$
\tau_{\mathrm{iner}} \approx \frac{a^{2}}{4 \nu} \text {. }
$$

To provide a numerical example, we take the channel dimensions to be $a=0.1$ $\mathrm{mm}$ and $L=10 \mathrm{~mm}$, and the liquid to be water at room temperature, i.e. $v=10^{-6}$ $\mathrm{m}^{2} \mathrm{~s}^{-1}$ and $c_{0}=1500 \mathrm{~m} \mathrm{~s}^{-1}$. The pressure propagation time becomes $L / c_{0} \approx 10 \mu \mathrm{s}$ and the inertial stopping time is $\tau_{\text {iner }} \approx 3$ ms, and the conditions $N_{1} \ll 1$ and $N_{2} \ll$ 1 for the analysis are thus fulfilled. We note that $\tau_{\text {iner }} \approx 3 \mathrm{~ms}$ for $a=0.1 \mathrm{~mm}$ combined with the $a^{2}$ scaling law implies, that for channels 10 times wider the inertial time scale approaches $1 \mathrm{~s}$, while 10 times more narrow leads to inertial time scales approaching the pressure propagation time scale. In Fig. 3 we show in detail how the axial velocity profile $v_{x}(r, t)$ develops in time from the initial removal of the driving pressure at $t=0$ in units of the characteristic stopping time $\tau_{\text {iner }}$ of eqn (14), for details of the calculation see ref. 3 and 4 . The figure shows how the velocity profile does indeed decay on a time scale given by $\tau_{\text {iner }}$.

\section{Molecular diffusion}

The handling of biological cells using acoustofluidics often involves the transfer of cells from one laminar buffer flow stream to another flowing in parallel. The acoustic forces are controlling the transfer of cells between the flow streams, while concentration gradients give rise to exchange of solutes between the buffers. In this section we study the latter.

To the extent that the solutes are not undergoing chemical reactions, they are conserved, and we can derive a continuity equation for the concentration field $c_{\alpha}(\boldsymbol{r}, t)$ for a given solute characterized by the index $\alpha$. The SI unit for $c_{\alpha}$ is $\mathrm{m}^{-3}$ or $\mathrm{M}=$ mol L $\mathrm{L}^{-1}$, where $1 \mathrm{M}=6.02 \times 10^{26}$ $\mathrm{m}^{-3}$. To derive the continuity equation for $c_{\alpha}$ we begin by stating the current density $\boldsymbol{J}_{\alpha}$ in analogy to the procedure for the buffer, Sec. IV in ref. 1,

$$
\boldsymbol{J}_{\alpha}=-D_{\alpha} \boldsymbol{\nabla} c_{\alpha}+c_{\alpha} \boldsymbol{v},
$$

where the first term on the right-hand side is Fick's first law for concentrationinduced solvent current density with solute diffusivity, $D_{\alpha}$, in the given solvent, and the second term represents the contribution from advection to the current density from the velocity field $\boldsymbol{v}$ of the buffer. The resulting advectiondiffusion equation, valid for weak solutions $c_{\alpha} \lesssim 0.1 \mathrm{M}$, is ${ }^{1,3}$

$$
\partial_{t} c_{\alpha}+(\boldsymbol{v} \cdot \nabla) c_{\alpha}=D_{\alpha} \nabla^{2} c_{\alpha}
$$

In the dilute limit the solutes are not affecting the velocity field of the solvent, so in the following the velocity field $v$ is assumed to be known from solving the flow problem separately. Moreover, for dilute solute concentrations the advection-diffusion equation is linear in $c_{\alpha}$, and all effects scales trivially with the concentration level, which therefore can be left out of the Buckingham P-matrix here,

$$
\mathbf{P}=\left(\begin{array}{ccccc} 
& a & L & v & D_{\alpha} \\
\mathrm{L} & 1 & 1 & 1 & 2 \\
\mathrm{~T} & 0 & 0 & -1 & -1
\end{array}\right) .
$$

The $\mathbf{P}$-matrix has $\mathscr{N}=\mathscr{D}-\mathscr{F}=4-2=2$ null vectors,

$$
\boldsymbol{\pi}_{1}=\left(\begin{array}{c}
1 \\
-1 \\
0 \\
0
\end{array}\right), \quad \boldsymbol{\pi}_{2}=\left(\begin{array}{c}
1 \\
0 \\
1 \\
-1
\end{array}\right),
$$

corresponding to the dimensionless groups or numbers

$$
N_{1}=\frac{a}{L}, \quad N_{2}=\frac{a v}{D_{\alpha}} .
$$

The first of these is the aspect ratio of the channel, and the second is the ratio of the axial advection speed relative to the transverse diffusion speed and is known as the Péclet number Pé

$$
P e ́=\frac{a v}{D_{\alpha}}
$$

Whether $P e ́$ is larger or smaller than unity is important for determining if advection or diffusion dominates. In this respect Pé 

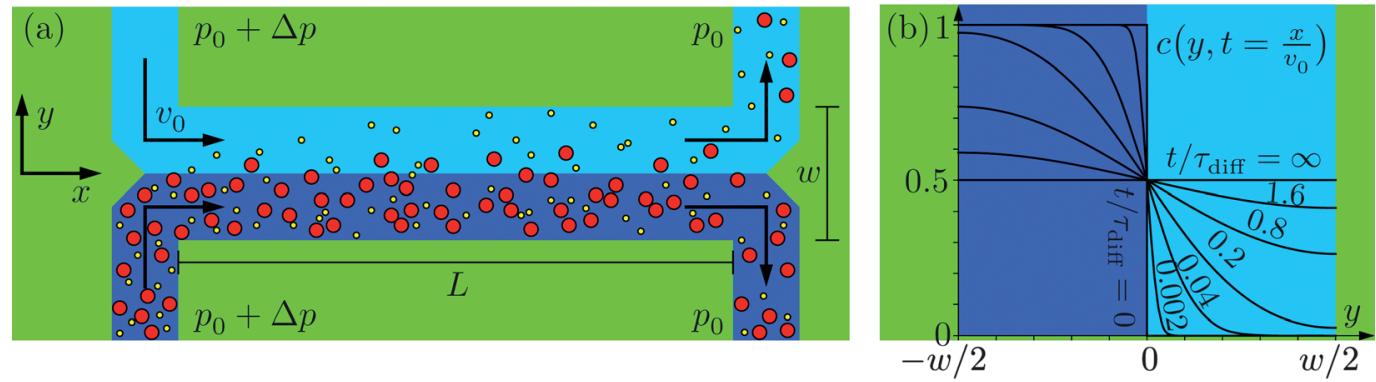

Fig. 4 (a) An $x y$-plane top view of a flat H-filter (height $h$ ) consisting of a central channel (length $L$ and width $w$ ) with two inlet channels to the left and two outlet channels to the right. A pure buffer liquid (light blue) and a buffer liquid (dark blue) containing big (red) and small (yellow) solutes are introduced via the top-left and bottom-left inlet, respectively, and form a pressure-driven, steady state, laminar flow with average velocity $v_{0}$. (b) Concentration profiles $c\left(y, t=x / v_{0}\right)$, in units of $c^{*}$, in the central channel as a function of the transverse direction $y$ at different positions $x$ along the channel. Adapted from ref. 3.

resembles $R e$, and interestingly, the latter can be written as $R e=a v / v$, showing that the Reynolds number is for momentum diffusion with diffusivity $\nu$ what the Péclet number is for molecular diffusion with diffusivity $D_{\alpha}$.

The molecular diffusivity for all small ions in water, such as $\mathrm{Na}^{+}$and $\mathrm{Cl}^{-}$, is around $2 \times 10^{-9} \mathrm{~m}^{2} \mathrm{~s}^{-1}$. For a typical microfluidic channel with $a \approx 0.1 \mathrm{~mm}$, this results in a threshold velocity $v=$ $D_{\alpha} / a \approx 20 \mu \mathrm{m} \mathrm{s}^{-1}$, above which advection effects become important. This is a relatively low velocity, so advection is expected to play a role for diffusion processes in microfluidic systems.

We now return to our problem of deriving the scaling laws for transverse diffusion between two parallel laminar buffer streams in a long, straight microfluidic channel of small height $h$ and large width $w$, such that $h \ll w$ as shown in Fig. 4(a). Let the channel have length $L$, and for simplicity let the two flow streams have the same width $w / 2$. One buffer is pure water (light blue), and the other is a dilute aqueous solution (dark blue), such that the viscosity and density of the two buffers are approximately equal. The average axial ( $x$-direction) flow speed in the channel is called $v_{0}$, and there is no velocity in the transverse direction ( $y$ direction). The steady-state advectiondiffusion problem thus becomes

$$
v_{0} \partial_{x} c_{\alpha}=D_{\alpha} \partial_{y}^{2} c_{\alpha},
$$

where we have assumed that the transverse concentration gradients are much larger than the axial. This can also be interpreted as the normal diffusion equation $\partial_{t} c_{\alpha}=D_{\alpha} \partial_{y}^{2} c_{\alpha}$, with "time" $t=x / v_{0}$. The solute concentration of the solution buffer is denoted $c^{*}$. As solutes begin to leave the solution buffer by diffusion as sketched in Fig. 4(b), the concentration at the interface (taken to be at $y=0$ ) between the two buffers remains at the value $c^{*} / 2$ for symmetry reasons. By inspection, it is straightforward to show that the solution to eqn (21) can be written in terms of the complementary error function $\operatorname{erfc}(s) \equiv \frac{2}{\sqrt{\pi}} \int_{s}^{\infty} \mathrm{e}^{-u^{2}} \mathrm{~d} u$,

$$
c(y, t>0)=\frac{1}{2} c^{*} \operatorname{erfc}\left(\frac{y}{\sqrt{4 D_{\alpha} t}}\right) \text {. }
$$

As $\operatorname{erfc}(s)$ is related to the Gauss distribution $\exp \left(-s^{2}\right)$, it follows that the time $\tau$ it takes to diffuse the length $\ell$ is roughly given by

$$
\iota^{2} \approx 2 D_{\alpha} \tau
$$

We can now easily derive the scaling law for mixing by diffusion between the two buffer streams of our problem. The transit or advection time, $\tau_{\mathrm{adv}}$, through the channel for a small group of solutes is given by $\tau_{\mathrm{adv}}=L / v_{0}$. The diffusion time $\tau_{\text {diff, } \alpha}$, for the same molecules to cover the transverse distance $w / 2$ and fill the pure water stream, is found by eqn (23) to be

$$
\tau_{\text {diff }, \alpha}=\frac{w^{2}}{8 D_{\alpha}} .
$$

The threshold for mixing is when $\tau_{\text {adv }}=$ $\tau_{\text {diff, } \alpha}$, and the corresponding flow velocity $v_{\alpha}$ obeys the scaling law

$$
v_{\alpha} \approx \frac{8 D_{\alpha} L}{w^{2}} .
$$

In the limit $v_{0} \ll v_{\alpha}$ or $P e_{\alpha} \ll 1$ the $\alpha$ solute mixes completely by diffusion, while $v_{0} \gg v_{\alpha}$ or $P e_{\alpha} \gg 1$ corresponds to advection with negligible spread by diffusion. Taking $L=10 \mathrm{~mm}, w=0.1$ $\mathrm{mm}$, and $D_{\alpha}=2 \times 10^{-9}$ results in the threshold velocity $v_{\alpha} \approx 10 \mathrm{~mm} \mathrm{~s}^{-1}$ which is a very convenient value as both larger and smaller flow velocities are easily set up experimentally, either by adjusting the driving pressure or the channel geometry through the factor $w^{2} / L$.

Given two solutes (index 1 and 2) with different diffusivities $D_{1}<D_{2}$, the H-filter can be used to a partial separation of the solutes as illustrated in Fig. 4(a) by the big red particles (index 1) and the small yellow particles (index 2). Given that $v_{1} \ll$ $v_{0} \ll v_{2}$ or equivalently $\tau_{\text {diff }, 2} \ll \tau_{\text {adv }} \ll$ $\tau_{\text {diff, } 1}$, the top-right output will contain a nearly $50 \%$ dilution of the original concentration of the small $\alpha=2$ particles and nearly zero concentration of the big $\alpha=1$ particles. Given the scaling law eqn (25), the H-filter is only efficient if the diffusivities $D_{1}$ and $D_{2}$ are sufficiently different.

The last scaling to mention relating to advection-diffusion is the one for the effective diffusivity $D_{\text {eff }}$ in Taylor dispersion, the flow-induced enhancement of dispersion of concentration fields. ${ }^{5-7}$ Consider a circular channel of radius $a$ initially containing a thin slab of solute which has a constant concentration $c^{*}$ inside a narrow axial region of width $\delta x \ll a$ and zero outside. If the flow velocity of the solvent is zero, the slab will broaden in time by diffusion governed by the diffusivity $D_{\alpha}$. If now a Poiseuille flow profile of average velocity $v_{0}$ is established, the slab will be stretched unevenly in the axial direction due to the nonuniform velocity profile, which is zero at the walls and maximum in the center. Consider a time step $\tau_{\text {diff }}$ long enough to 
Table 1 List of values of material parameters at $20^{\circ} \mathrm{C}$ for typical liquids [water (wa), $\mathrm{NaCl}$ solution (scs), percoll (pc), glycerol (gl)] and solids [pyrex (PY), polystyrene (PS), polymethacrylate (PM), melamine resin (MR), a representative biological cell (Cell)] used in microchannel acoustophoresis

\begin{tabular}{lcccc}
\hline Material & Density $\rho\left[\mathrm{kg} \mathrm{m}^{-3}\right]$ & Compressibility $\kappa[1 / \mathrm{TPa}]$ & Longitudinal speed of sound $c\left[\mathrm{~m} \mathrm{~s}^{-1}\right]$ & Viscosity $\eta[\mathrm{mPa} \cdot \mathrm{s}]$ \\
\hline $\mathrm{wa}^{a}$ & 998.2 & 456 & 1482 & 1.002 \\
$\mathrm{scs}^{b}$ & 1071 & 365 & 1599 & 1.170 \\
$\mathrm{pc}^{c}$ & 1130 & 390 & 1907 & 100 \\
$\mathrm{gl}^{a}$ & 1261 & 219 & 5674 & 1412 \\
$\mathrm{PY}^{a}$ & 2230 & 27.8 & 2350 & - \\
$\mathrm{PS}^{c}$ & 1050 & 172 & 2380 & - \\
$\mathrm{PM}^{c}$ & 1190 & 148 & 3132 & - \\
$\mathrm{MR}^{c}$ & 1510 & 67.5 & 1500 & - \\
$\mathrm{Cell}^{d}$ & 1100 & 400 &
\end{tabular}

${ }^{a}$ From ref. $11{ }^{b}$ Sodium chloride solution of salinity $S=0.1$, from ref. $12 .{ }^{c}$ From Sigma-Aldrich Production GmbH and Fluka data sheets. ${ }^{d}$ From ref. 13 and 14.

imply a radial diffusion distance squared of $(\Delta r)^{2}=(a / 2)^{2}=a^{2} / 4$ which samples the radial gradient of the axial velocity well. During the time $\tau_{\text {diff }}$ the one-dimensional axial diffusion distance squared due solely to molecular diffusion is half of that in the two-dimensional radial direction $(\Delta x)^{2}=(\Delta r)^{2} / 2=a^{2} / 8$. However, the transverse diffusion implies an additional axial diffusion step of the order $\Delta \tilde{x} \approx$ $v_{0} \tau_{\text {diff }}$, where $\tau_{\text {diff }}=(\Delta x)^{2} /\left(2 D_{\alpha}\right)$. Thus the total axial diffusion distance squared is. $(\Delta x)^{2}+(\Delta \tilde{x})^{2}=\left[1+\frac{1}{32}\left(v_{0} a / D_{\alpha}\right)^{2}\right](\Delta x)^{2}$ As $D_{\alpha}=(\Delta x)^{2} /\left(2 \tau_{\text {diff }}\right)$, it is natural to define the effective dispersion as $D_{\text {eff }}=\left[(\Delta x)^{2}+(\Delta \tilde{x})^{2}\right] /\left(2 \tau_{\text {diff }}\right)$, and we arrive the scaling law for $D_{\text {eff }}$,

$$
\begin{aligned}
& D_{\mathrm{eff}} \approx \frac{(\Delta x)^{2}+(\Delta \tilde{x})^{2}}{(\Delta x)^{2}} D_{\alpha} \\
& =\left[1+\frac{1}{32}(P e ́)^{2}\right] D_{\alpha} .
\end{aligned}
$$

As before the numerical pre-factor in the scaling law is not accurate, and the correct expressions for the effective diffusivity $\operatorname{are}^{5-7}$

$$
\begin{aligned}
D_{\text {eff }}= & {\left[1+\frac{1}{48}(P e ́)^{2}\right] D_{\alpha}, } \\
& \text { circular channel, } \\
D_{\text {eff }}= & {\left[1+\frac{2}{105}(P e ́)^{2}\right] D_{\alpha}, } \\
& \text { parallel-plate channel, }
\end{aligned}
$$

The scaling law shows that the effective axial diffusivity is much larger than the molecular diffusivity and scales like the Péclet number to the power two, $D_{\text {eff }} \propto$ $(P e ́)^{2} D_{\alpha}$, for $P e ́$. We have seen that
$P e ́ \approx 1$ for $v_{0} \approx 20 \mu \mathrm{m} \mathrm{s}^{-1}$ for typical acoustofluidic channel with $a \approx 0.1 \mathrm{~mm}$, so in practice we often have $P e ́$ $\gg 1$.

\section{Ultrasound acoustics}

The basics of ultrasound acoustics for microsystems acoustophoresis is reviewed in earlier parts of the Tutorial Series: In Part 2 the ultrasound modes are treated in simplified microfluidic channels, ${ }^{8}$ Part 3 includes the continuum mechanics of the elastic walls; ${ }^{9}$ and Part 4 treats the piezo electric transducers generating the ultrasound waves. ${ }^{10}$ It is difficult to derive scaling laws for the complete acoustical system consisting of the elastic chip containing the microfluidic channel and the attached piezo transducer, because of the many parameters that have identical physical dimensions. The geometry of the full system contains a number of length scales characterizing each sub-system, and likewise each material has its own acoustic parameters, see Table 1 .

The order of magnitude of the speed of sound $c_{j}$ of a given material $j$ is given by the density $\rho_{j}$ and bulk modulus $K_{j}=-V \partial p / \partial V$ (the inverse of the compressibility $\kappa_{j}$ ) from the relative changes in volume $V$ for a change in pressure $p$ as

$$
c_{j} \propto \sqrt{\frac{K_{j}}{\rho_{j}}} .
$$

The density of liquids and solids is typically in the range $10^{3}-10^{4} \mathrm{~kg} \mathrm{~m}^{-3}$, the bulk modules in the range $2-200 \mathrm{GPa}$, and the speed of sound is in the range $1-10 \mathrm{~km} \mathrm{~s}^{-1}$. For solids, the scaling law eqn (28) is complicated by the existence of more than one speed of sound depending of the polarization of the sound wave (longitudinal or transverse) and for crystal structures also the propagation direction relative to the crystal axes.

\section{A. Resonances}

We limit the following scaling analysis of acoustic resonances to the case dominated by those in water, with the sound speed $c_{\mathrm{wa}}=1500 \mathrm{~m} \mathrm{~s}^{-1}$, inside a rectangular water-filled channel of length $L$, width $w$, and height $h$, surrounded by infinitely hard walls. In ref. 8 the resonance frequencies of this system are found to be

$$
\begin{aligned}
& f_{n_{x}, n_{y}, n_{z}}=\frac{c_{\mathrm{wa}}}{2} \sqrt{\frac{n_{x}^{2}}{L^{2}}+\frac{n_{y}^{2}}{w^{2}}+\frac{n_{z}^{2}}{h^{2}}}, \\
& \text { with } n_{x}, n_{y}, n_{z}=0,1,2,3,4, \ldots
\end{aligned}
$$

We study a long, flat channel for which $h \lesssim w \ll L$, and for the numerical estimates we take $h=0.15 \mathrm{~mm}, w=0.4 \mathrm{~mm}$, and $L=40 \mathrm{~mm}$, corresponding to the channels used in ref. 15 and 16 . To establish a resonance with a half-wavelength or more in the $z$-direction, a frequency of at least $f_{0,0,1}=c_{\text {wa }} /(2 h) \approx$ $5 \mathrm{MHz}$ is needed. For frequencies below this cut-off, only waves in the axial and wide transverse directions can be excited. The corresponding resonances are

$$
f_{n_{x}, n_{y}, 0}=\frac{c_{\mathrm{wa}}}{2} \sqrt{\frac{n_{x}^{2}}{L^{2}}+\frac{n_{y}^{2}}{w^{2}}} .
$$

In the classic acoustophoretic separation device, ${ }^{8,17-19}$ one seeks to work with the transverse half-wavelength resonance $f_{0,1,0}=c_{\mathrm{wa}} /(2 w) \approx 2 \mathrm{MHz}$. The spacing 
$\Delta f\left(n_{x}\right)$ between modes $f_{n_{x}, 1,0}$ is estimated by $\Delta f\left(n_{x}\right) \approx\left[\partial f_{n_{x}, 1,0} / \partial n_{x}\right] \Delta n_{x}$ with $\Delta n_{x}=1$,

$$
\Delta f\left(n_{x}\right)=\frac{w^{2}}{L^{2}} \frac{n_{x}}{\sqrt{1+\frac{w^{2}}{L^{2}} n_{x}^{2}}} f_{0,1,0} .
$$

For the lower modes with $n_{x} \lesssim L / w \approx$ 100 , we find

$$
\Delta f\left(n_{x}\right) \approx \frac{w^{2}}{L^{2}} f_{0,1,0} n_{x}, \quad \text { for } n_{x} \lesssim \frac{L}{w} .
$$

For $n_{x} \approx 20$ we find $\Delta f\left(n_{x}\right) \approx 4 \mathrm{kHz}$. We note that the longer the channel, the smaller the separation of resonance frequencies and the more resonances are contained within a given frequency bandwidth. It is relevant to compare the mode separation to the width $\delta f$ of the resonances given by the Q-value (not to be confused with the flow rate $Q$ ). Experimentally it is found that $Q \approx 500$ is a typical Q-value, ${ }^{9,15}$ and consequently

$$
\delta f \approx \frac{1}{Q} f_{0,1,0} \approx 4 \mathrm{kHz} .
$$

Thus the mode separation $\Delta f$ is comparable to the mode width $\delta f$, and the resonances are expected to be marginally separated. This is also seen experimentally ${ }^{15,16}$ as discussed in Part 7 and 8 of the Tutorial Series. ${ }^{19,20}$ For lower Q-values the resonances overlap more, resulting in broad-band, blurred axial waves, while maintaining the well-defined transverse half-wave. Quantitatively, the scaling law is again not accurate; the experimentally observed values are $\Delta f \approx \delta f \approx 10 \mathrm{kHz}$.

\section{B. Energy density}

As shown in the following section, the acoustic force in acoustophoretic particle handling scales like the acoustic energy density $E_{\mathrm{ac}}$ of the standing ultrasound wave to the power one. It is therefore of interest to study the scaling law for $E_{\mathrm{ac}}$. An acoustic wave can be thought of as a harmonic oscillator, where the energy during an oscillation period changes between kinetic energy of the liquid and pressure-related potential energy. We therefore expect the scaling relation $E_{\mathrm{ac}} \propto$ $p_{\mathrm{a}}^{2}$, where $p_{\mathrm{a}}$ is the amplitude of the standing pressure wave. Since both $E_{\mathrm{ac}}$ and $p_{\mathrm{a}}$ have the SI unit $\mathrm{J} \mathrm{m}^{-3}=\mathrm{Pa}$, dimensional analysis requires that we must divide $p_{\mathrm{a}}^{2}$ by a quantity of unit $\mathrm{Pa}$ to obtain $E_{\mathrm{ac}}$. The only relevant quantity available in the problem is the bulk modulus of eqn (28), $K_{0}=\rho_{0} c_{0}^{2}$, where index " 0 " refer to the liquid. Hence, we have the scaling law $E_{\mathrm{ac}} \propto p_{\mathrm{a}}^{2} / K_{0}$, and an exact analysis brings in a factor of $\frac{1}{4}$,

$$
E_{\mathrm{ac}}=\frac{p_{\mathrm{a}}^{2}}{4 K_{0}}=\frac{p_{\mathrm{a}}^{2}}{4 \rho_{0} c_{0}^{2}}=\frac{1}{4} \kappa_{0} p_{\mathrm{a}}^{2},
$$

where $\kappa_{0}=1 / K_{0}$ is the compressibility of the liquid.

In Part 4 of the Tutorial Series, ${ }^{10}$ the basic theory of piezoelectric actuation of ultrasonic resonances in water-filled silicon/glass microchannels is presented. It is shown that a linear relation exists between the applied peak-to-peak voltage $U_{\mathrm{pp}}$ of the piezo transducer responsible for exciting the ultrasonic resonance and the induced acoustic pressure amplitude $p_{\mathrm{a}}$,

$$
P_{\mathrm{a}} \propto U_{\mathrm{pp}}
$$

From eqn (34), $E_{\mathrm{ac}}$ thus scales with the square of $U_{\mathrm{pp}}$,

$$
E_{\mathrm{ac}} \propto U_{\mathrm{pp}}^{2} .
$$

As summarized in Part 7 of the Tutorial Series, ${ }^{19}$ this scaling law was tested in ref. 15 by plotting the values of $E_{\text {ac }}$ extracted by a particle-tracking method versus the applied piezo-transducer voltage $U_{\mathrm{pp}}$. In typical acoustophoretic experiments in water-filled silicon/glass chips $E_{\mathrm{ac}}=$ $10-100 \mathrm{~J} \mathrm{~m}^{-3}$.

\section{Acoustophoresis}

We end this Tutorial with a discussion of the scaling laws for acoustophoretic particle handling. It includes dimensional analysis of the magnitude of the acoustic radiation force, the Stokes drag force, transverse acoustophoretic focusing time, axial advection time, vertical sedimentation time, and the acoustic streaming.

\section{A. The acoustic radiation force}

The fundamental force behind acoustophoretic particle handling is $F_{\text {rad }}$, the acoustic radiation force, ${ }^{12,21,22}$ which is due to scattering of ultrasound waves on the particle, as treated in detail in Part 7 of the Tutorial Series. ${ }^{19}$ The scaling law for $F_{\text {rad }}$ can be derived using dimensional analysis for a spherical particle of radius $a$, density $\rho_{\mathrm{p}}$ and compressibility $\kappa_{\mathrm{p}}$ suspended in a fluid of density $\rho_{0}$, compressibility $\kappa_{0}$, and speed of sound $c_{0}$ under the influence of a given "incoming" sinusoidal, standing pressure wave $p_{\text {in }}$,

$$
p_{\text {in }}(y, t)=p_{\text {a }} \cos (k y) \cos (\omega t),
$$

of wavelength $\lambda$, wavenumber $k=2 \pi / \lambda$, frequency $f$, and angular frequency $\omega=$ $2 \pi f=k c_{0}$. We treat the small-particle limit $k a=2 \pi a / \lambda \ll 1$, and note that for large particles, $a \gtrsim 3 \mu \mathrm{m}$, viscous effects of the fluid is negligible for ultrasound waves in water. ${ }^{12,19}$ The pressure wave $p_{\text {in }}$ is associated velocity wave ${ }^{19}$ $\boldsymbol{v}_{\text {in }} \propto \nabla p_{\text {in. }}$.

If the relative compressibility $\tilde{\kappa}=\kappa_{\mathrm{p}} / \kappa_{0}$ deviates from unity, the particle acts as a point scatterer for pressure waves, $p_{\text {sc }} \propto$ $(\tilde{\kappa}-1) p_{\text {in }}$. Similarly, if the relative density $\tilde{\rho}=\rho_{\mathrm{p}} / \rho_{0}$ deviates from unity, it acts as a point scatter for velocity waves given by $v_{\mathrm{sc}}=(\tilde{\rho}-1) v_{\mathrm{in}}$. For weak (first-order) scattering, the monopole and dipole scattering do not mix. Because the time average of the harmonically oscillating standing pressure and velocity waves is zero, $\left\langle p_{\text {in }}\right\rangle=0$ and $\left\langle v_{\text {in }}\right\rangle=0$, the radiation force must be a second-order effect for which the time average does not vanish, $\left\langle\cos ^{2}(\omega t)\right\rangle=1 / 2$. Two types of secondorder terms are linear in the scattered fields and thus dominating, namely $\left\langle p_{\mathrm{sc}} p_{\text {in }}\right\rangle \propto(\tilde{\kappa}-1) \kappa_{0}\left\langle p_{\text {in }}^{2}\right\rangle$ and $\left\langle v_{\mathrm{sc}} v_{\text {in }}\right\rangle \propto$ $(\tilde{\rho}-1) \rho_{0}\left\langle v_{\mathrm{in}}^{2}\right\rangle$, where the pre-factors $\kappa_{0}$ and $\rho_{0}$ are inserted to give the two terms the unit of energy density. The two acoustic energy densities must be related to the volume taken up by the particle in the liquid, so a factor $\frac{4}{3} \pi a^{3}$ must be included, resulting in two terms of dimension energy. We now need a last factor of unit $\mathrm{m}^{-1}$ to obtain the unit $\mathrm{N}$ for force. This is provided by the wave number $k$, the argument being that firstorder scattering is proportional to the (small) scattering parameter $k a \ll 1$. Equivalently, the wavenumber comes about from the gradient operator acting on the sinusoidal functions $\left\langle p_{\text {in }}^{2}\right\rangle \propto$ $\cos ^{2}(k y)$ and $\left\langle v_{\text {in }}^{2}\right\rangle \propto \sin ^{2}(k y)$. Here $v_{\text {in }} \propto$ $\sin (k y)$ because of the Stokes equation $\rho_{0} \partial_{t} v_{\text {in }}=-\partial_{y} p_{\text {in }}$. The resulting scaling law for the radiation force is then

$$
\begin{aligned}
\boldsymbol{F}^{\mathrm{rad}}= & -\frac{4}{3} \pi a^{3} \nabla\left[C_{1}(\tilde{\kappa}-1) \kappa_{0}\left\langle p_{\text {in }}^{2}\right\rangle\right. \\
& \left.+C_{2}(\tilde{p}-1) \rho_{0}\left\langle v_{\text {in }}^{2}\right\rangle\right]
\end{aligned}
$$


where $C_{1}$ and $C_{4}$ are dimensionless constants. Detailed treatment gives $\quad C_{1}=-\frac{1}{2} \quad$ and $\quad C_{2}=\frac{1}{2} \frac{3}{2 \tilde{\rho}+1}$. Carrying out the differentiation and collecting terms, we end with the classic result $^{21,22}$ for the standing cosine pressure wave $p_{\text {in }}$ of eqn (37) between channel walls at $y=0$ and at $y=w$,

$$
\begin{gathered}
F_{\mathrm{rad}}=4 \pi \Phi(\tilde{\kappa}, \tilde{\rho}) k a^{3} E_{\mathrm{ac}} \sin (2 k y), \\
\Phi(\tilde{\kappa}, \tilde{\rho})=\frac{1}{3}\left[\frac{5 \tilde{\rho}-2}{2 \tilde{\rho}+1}-\tilde{\kappa}\right],
\end{gathered}
$$

where $\Phi(\tilde{\kappa}, \tilde{\rho})$ is the acoustophoretic contrast factor. For the simple standing half-wavelength, the wavenumber becomes $k=\pi / w$, and the radiation force focuses all particles with $\Phi>0$ at the central plane at $y=w / 2$. For $\Phi<0$ the particles are focused at the walls. ${ }^{17-19}$

\section{B. Particle motion in microfluidics}

Viscosity dominates in microfluidics in general and for the motion of micrometersized particles in particular. The scaling law for the acceleration time $\tau_{\text {acc }}$ of a particle of radius $a$ and density $\rho_{\mathrm{p}}$ moving in a liquid of viscosity $\eta$ is easily found by dimensional analysis,

$$
\tau_{\mathrm{acc}} \approx \frac{\rho_{\mathrm{p}} a^{2}}{\eta}=\tilde{\rho} \frac{a^{2}}{\nu} .
$$

For a $5 \mu \mathrm{m}$ diameter particle in water $\tau_{\text {acc }}$ $\approx \tilde{\rho} \times 3 \mu \mathrm{s}$, a time-scale so short that the usual assumption about instantaneous steady-state motion of microparticles is justified. The frictional force responsible for this steady-state motion is the Stokes drag force, $F_{\text {drag. }}$. The scaling law for $F_{\text {drag }}$ for a sphere of radius $a$ moving with the velocity $v$ through a fluid of viscosity $\eta$ is derived from multiplying the surface area $4 \pi a^{2}$ with the viscous stress $\eta(v / a)$, so that $F_{\text {drag }} \approx 4 \pi \eta a v$. An exact treatment ${ }^{3,23}$ yields

$$
\boldsymbol{F}_{\text {drag }}=-6 \pi a \eta \boldsymbol{v}
$$

The time $\tau_{\text {foc }}$ it takes to focus particles with $\Phi>0$ at the central plane $y=w / 2$ is found by balancing the radiation force and the Stokes drag force,

$$
F_{\text {rad }}(y)=F_{\text {drag }}=6 \pi a \eta \frac{\mathrm{d} y}{\mathrm{~d} t} .
$$

This differential equation can be solved analytically ${ }^{15,19,24}$ for $y(t)$, but here we just use dimensional analysis to obtain the scaling law for $\tau_{\text {foc. }}$. Since $\left[E_{\mathrm{ac}}\right]=\mathrm{Pa}$ and $[\eta]=$ Pa s, we immediately obtain $\tau_{\text {foc }} \propto$ $\eta / E_{\mathrm{ac}}$. For the standing half wave $\lambda=2 w$, and we only have two length scales in the problem, $a$ and $w$. So how does the aspect ratio $a / w$ enter in the scaling for $\tau_{\text {foc }}$ ? As $F_{\text {rad }} \propto a^{3}$ and $F_{\text {drag }} \propto a$, we are left with a factor $a^{2}$, so the aspect ratio must enter to the power two, $a^{2} / w^{2}$, and the smaller the particle, the longer the focusing takes, hence $\tau_{\text {foc }} \propto(w / a)^{2} \eta / E_{\mathrm{ac}}$. Solving eqn (42) for a particle moving from $y=0$ to $y=$ $w / 2$, and with $\sin (\pi y / w)$ approximated by its average value $2 / \pi$, we obtain

$$
\tau_{\mathrm{foc}} \approx \frac{3}{8 \Phi} \frac{w^{2}}{a^{2}} \frac{\eta}{E_{\mathrm{ac}}} .
$$

The exact analytical solution only results in a weak logarithmic correction to this expression. For $3 /(8 \Phi) \approx 1, a=5 \mu \mathrm{m}, w=$ $0.4 \mathrm{~mm}, \eta=1 \mathrm{mPas}$, and $E_{\mathrm{ac}}=100 \mathrm{~J} \mathrm{~m}^{-3}$ we obtain the fairly short focus time of $\tau_{\text {foc }}=60 \mathrm{~ms}$. This is a very convenient value, which by virtue of the scaling law can easily be adjusted in a wide range to optimize devices for acoustophoretic handling.

\section{Advection and sedimentation}

An important use of the acoustic radiation force is to obtain fractionation of particles in flow-through mode of a given microchannel. For an average axial flow velocity $v$ in a channel of length $L$, the advection time $\tau_{\text {adv }}$ for passing through the channel by advection, is given by

$$
\tau_{\mathrm{adv}}=\frac{L}{v}
$$

For an aqueous solution of two types of particles with radii $a_{1} \ll a_{2}$, it is fairly easy to adjust the parameter to obtain $\tau_{\text {foc }}\left(a_{2}\right)$ $\ll \tau_{\text {adv }} \ll \tau_{\text {foc }}\left(a_{1}\right)$, in which case the large $a_{2}$-radius particles are focused at the center plane well before reaching the outlet, while the small $a_{1}$-radius particles have hardly moved transversely. The closer the ratio $a_{1} / a_{2}$ is to unity, the less sensitive is this separation method. The most sensitive mode is the binary separation obtained when $\Phi_{1}$ and $\Phi_{2}$ have opposite signs, as in the seminal microchannel acoustophoresis work ref. 17 and 18.

Similarly, it is important to determine the time $\tau_{\text {sed }}$ it takes a given type of particle to sediment in a microfluidic channel. The scaling law for $\tau_{\text {sed }}$ is obtained by equating the buoyancy force $\quad F_{\text {buoy }}=(\tilde{\rho}-1) \frac{4}{3} \pi a^{3} \rho_{0} g \quad$ with Stokes drag $F_{\text {drag }}=6 \pi \eta a\left(h / \tau_{\text {sed }}\right)$, where $h$ is the sedimentation distance (height of the channel). The result is

$$
\tau_{\text {sed }}=\frac{9}{2(\tilde{\rho}-1)} \frac{\nu h}{a^{2} g} .
$$

Acoustophoretic focusing requires $\tau_{\text {foc }} \ll$ $\tau_{\text {sed }}$, from which the scaling law follows,

$$
\frac{(\tilde{\rho}-1)}{12 \Phi} \frac{w^{2}}{h^{2}} \rho_{0} g h \ll E_{\mathrm{ac}} .
$$

It turns out not to be a severe constraint. For $\tilde{\rho}=2, h=0.15 \mathrm{~mm}, w=0.4 \mathrm{~mm}$, we obtain $E_{\mathrm{ac}} \gg 1 \mathrm{~J} \mathrm{~m}^{-3}$.

\section{Acoustic streaming}

Acoustic streaming is a second-order time-averaged phenomenon, in which the fluid is set in motion by the acoustic wave through momentum exchange in the viscous boundary layer close to the walls. Streaming often shows up as steady vortices. ${ }^{19,25}$ For an acoustic field oscillating with the angular frequency $\omega$, the time scale for carrying information about the changing bulk field is given by $1 / \omega$. The distance $\delta$ away from the wall over which the fluid has time to adjust to one flow direction is given by $^{23,26,27}$

$$
\delta=\sqrt{\frac{2 \nu}{\omega}} \approx 0.6 \mu \mathrm{m},
$$

in analogy with eqn (23), and where the value is for $1 \mathrm{MHz}$ ultrasound in water at room temperature. The length scale $\delta$ is the width of the viscous boundary layer surrounding any wall. Interestingly, the boundary-layer does not enter the scaling law for the magnitude of the streaming velocity $v_{\text {str }}$. Since streaming is a secondorder effect, $v_{\text {str }} \propto v_{\mathrm{a}}^{2}$, where $v_{\mathrm{a}}$ is the amplitude of $v_{\text {in }}$, and the only other velocity in the problem is the speed of sound in the fluid, so

$$
v_{\mathrm{str}}=\Psi \frac{v_{\mathrm{a}}^{2}}{c_{0}} .
$$

Here, $\Psi$ is a geometry-dependent factor of order unity, e.g. $\Psi=3 / 8$ for a standing wave parallel to a planar wall. A particle kept fixed in an acoustic streaming field $v_{\text {str }}$ is subject to an acoustic streaming force $F_{\text {str }}$ from viscous drag of magnitude 


$$
F_{\text {str }}=6 \pi \eta a v_{\text {str }}=6 \pi \eta a \Psi \frac{v_{\mathrm{a}}^{2}}{c_{0}} .
$$

We can now obtain an estimate for the critical particle radius $a_{\mathrm{c}}$, below which the radiation force $F_{\text {rad }} \propto a^{3}$ no longer dominates and the particle motion is governed by the less understood and less controlled advection by acoustic streaming. If we assume that the particle is kept in place by a force balance between the radiation force and the force from acoustic streaming, $F_{\text {rad }}=F_{\text {str }}$, then expressions (39a) and (40) for the forces lead to

$$
2 \pi a_{c}^{3} k \rho_{0} v_{\mathrm{a}}^{2} \Phi \approx 6 \pi \eta a_{c} \Psi \frac{v_{\mathrm{a}}^{2}}{c_{0}},
$$

from which we obtain the scaling law for the critical particle radius $a_{\mathrm{c}}$ or diameter $d_{\mathrm{c}}$,

$$
\begin{gathered}
a_{c}=\sqrt{\frac{3 \Psi}{2 \Phi}} \delta \approx 1 \mu \mathrm{m}, \\
d_{c}=\sqrt{6 \frac{\Psi}{\Phi}} \delta \approx 2 \mu \mathrm{m},
\end{gathered}
$$

given a numerical pre-factor of the order of unity.

\section{Concluding remarks}

We have discussed various scaling laws in the broad context of acoustofluidics acoustophoresis, covering the topics of laminar flow in microfluidic channels, molecular diffusion in laminar flow streams, ultrasound acoustics, and motion of microparticles under influence of different forces. By the combination of the scaling laws with the example values provided in the text, it is possible to obtain a fair estimate of which values must be chosen for the design parameters for a given acoustophoretic device.

\section{Acknowledgements}

This work was supported by the Danish Council for Independent Research, Technology and Production Sciences, Grant No. 274-09-0342.

\section{References}

1 H. Bruus, Lab Chip, 2011, 11, 3742-3751.

2 E. Buckingham, Phys. Rev., 1914, 4, 345376.

3 H. Bruus, Theoretical Microfluidics, Oxford University Press, Oxford, 2008.

4 G. K. Batchelor, An Introduction to Fluid Dynamics, Cambridge University Press, Cambridge, 1967.

5 G. I. Taylor, Proc. R. Soc. London, Ser. A, 1953, 219, 186.

6 R. Aris, Proc. R. Soc. London, Ser. A, 1956, 235, 67-77.

7 S. Vedel and H. Bruus, J. Fluid Mech., 2012, 691, 95-122.

8 H. Bruus, Lab Chip, 2012, 12, 20-28.

9 J. Dual and T. Schwarz, Lab Chip, 2012, 12, 244-252.

10 J. Dual and D. Möller, Lab Chip, 2012, 12, 506-514.
11 C. Product, CRC Handbook of Chemistry and Physics, Taylor and Francis Group, LLC, 90th edn, 2010.

12 M. Settnes and H. Bruus, Phys. Rev. E: Stat., Nonlinear, Soft Matter Phys., 2012, 85, 016327.

13 M. Godin, A. K. Bryan, T. P. Burg, K. Babcock and S. R. Manalis, Appl. Phys. Lett., 2007, 91, 123121.

14 P. Augustsson, R. Barnkob, C. Grenvall, T. Deierborg, P. Brundin, H. Bruus and T. Laurell, Proc. 14th MicroTAS, 3-7 October 2010, Groningen, The Netherlands, 2010, pp. 1337-39.

15 R. Barnkob, P. Augustsson, T. Laurell and H. Bruus, Lab Chip, 2010, 10, 563-570.

16 P. Augustsson, R. Barnkob, S. T. Wereley, H. Bruus and T. Laurell, Lab Chip, 2011, 11, 4152-4164.

17 F. Petersson, A. Nilsson, C. Holm, H. Jönsson and T. Laurell, Analyst, 2004, 129, 938-43.

18 F. Petersson, A. Nilsson, C. Holm, H. Jönsson and T. Laurell, Lab Chip, 2005, 5, 20-2.

19 H. Bruus, Lab Chip, 2012, 12, 1014.

20 A. Lenshof, C. Magnusson and T. Laurell, Lab Chip, 2012, DOI: 10.1039/ C2LC21256K.

$21 \mathrm{~K}$. Yosioka and Y. Kawasima, Acustica, 1955, 5, 167-173.

22 L. P. Gorkov, Soviet Physics - Doklady, 1962, 6, 773-775.

23 L. D. Landau and E. M. Lifshitz, Fluid Mechanics, Pergamon Press, Oxford, 2nd edn, 1993, vol. 6, Course of Theoretical Physics.

24 M. Kumar, D. Feke and J. Belovich, Biotechnol. Bioeng., 2005, 89, 129-137.

25 S. M. Hagsäter, T. G. Jensen, H. Bruus and J. P. Kutter, Lab Chip, 2007, 7, 1336-1344.

26 L. Rayleigh, Philos. Trans. R. Soc. London, $1884,175,1-21$.

27 S. Sadhal, Lab Chip, 2011, in press, (Tutorial Part 13). 